\title{
Controle biológico natural de pulgões (Hemiptera: Aphididae) em lavoura de trigo por parasitóides (Hymenoptera, Aphidiinae), no município de Medianeira, PR, Brasil
}

\section{Natural biological control of aphids (Hemiptera: Aphididae) in a wheat field by parasitoids (Hymenoptera, Aphidiinae) in Medianeira, PR, Brazil}

\author{
Luis Francisco Angeli Alves ${ }^{1 *}$; Tânia M.V. Prestes ${ }^{2}$; Agostinho Zanini²; \\ Maria F. Dalmolin²; Ayres de O. Menezes $\mathrm{Jr}^{3}$
}

\section{Resumo}

\begin{abstract}
Os pulgões são um dos principais problemas fitossanitários da cultura do trigo no sul do Brasil, sendo alvo de um programa de controle biológico por parasitóides implantado no Brasil a partir da década de 1970. A despeito do sucesso inicial rapidamente obtido não há na região oeste do Paraná nenhum estudo recente avaliando a situação atual do controle biológico dos pulgões, sendo este o objetivo do presente trabalho. Foram realizados levantamentos semanais em uma lavoura comercial de trigo em Medianera, PR, sendo os pulgões identificados e quando parasitados, analisados até a emergência do parasitóide. A população de pulgões não alcançou a média de um inseto/afilho, com a predominância de Rhopalosiphum padi (53\%), seguido de Sitobion avenae e R. maidis (19,5\%) e Metapophium dirhodum (8\%), surgindo principalmente a partir da fase de alongamento do trigo. Os parasitóides foram observados a partir da época de floração do trigo (cerca de três meses após o plantio), sendo identificadas apenas duas espécies de braconídeos Lysiphlebus testaceipes (97,3\%) e Diaretiella rapae (2,7\%), cujo crescimento populacional acompanhou a população de pulgões, seguindo a mesma tendência após a redução do número de pulgões no campo. Palavras-chave: Manejo integrado de pragas, dinâmica populacional, microhimenópteros
\end{abstract}

\begin{abstract}
Aphids constitute one of the main phytosanitary problems in wheat crops in southern Brazil, and are the target of a biological control program with parasitoids implemented in Brazil since the 1970's. In spite of the initial success quickly achieved, no recent study exists for the west region of the State of Paraná that evaluates the current status of aphid biological control; therefore, this was the object of the present work. Weekly surveys were conducted in a commercial wheat crop in Medianeira, PR. The aphids were identified and, when parasitization was verified, they were analyzed until emergence of the parasitoid. The population of aphids did not reach the mean value of 1 insect/tiller, with predominance of Rhopalosiphum padi (53\%), followed by Sitobium avenae and R. maidis (19.5\%) and Metopolophium dirhodum (8\%), which were verified mostly from the wheat elongation stage. The parasitoids were observed starting at the wheat flowering season (about 3 months after planting), and only two species of braconids, Lysiphlebus testaceipes (97.3\%) and Diaeretiella rapae (2.7\%), were identified, whose population growth followed the aphids population of, keeping the same trend after the number of aphids in the field was reduced.

Key words: Integrated pest managent, populational dinamic, microhymenoptera
\end{abstract}

1 Centro de Ciências Biológicas e da Saúde, Universidade Estadual do Oeste do Paraná, Campus de Cascavel PR. Laboratório de

Zoologia, Rua Universitária, 2069, Cascavel, PR, CEP: 85819-110 e-mail: 1faalves@unioeste.br

2 Centro Federal de Educação Tecnológica do Paraná, Caixa postal 391, 85884 000, Medianeira PR

3 Departamento de Agronomia, Universidade Estadual de Londrina, Londrina PR

* Autor para correspondência.

Recebido para publicação 19/10/04 Aprovado em 15/04/05 


\section{Introdução}

Os pulgões (Hemiptera: Aphididae) foram o principal problema fitossanitário no cultivo de cereais de inverno na década de 70 no sul do Brasil (SALVADORI; SALLES, 2002). A capacidade de dano desses insetos é elevada devido ao alto potencial biótico e toxidez da saliva para as plantas. Em ataques intensos, nas fases de emergência ao perfilhamento do trigo, em geral, levam as plantas à morte (GASSEN, 1984; SALVADORI; TONET, 2001).

As principais espécies de pulgões consideradas importantes como pragas sugadoras da cultura do trigo nos estados do Rio Grande do Sul, Paraná, São Paulo, Minas Gerais e Mato Grosso do Sul são Metopolophium dirhodum, Sitobion avenae, Rhopalosiphum padi, Schizaphis graminum e $R$. maidis. No entanto, estes insetos possuem importantes agentes de supressão populacional, como predadores, entomopatógenos e parasitóides, sendo estes últimos considerados os mais específicos e eficientes (SALVADORI; TONET, 2001).

Diante da problemática da praga e do ineficiente e oneroso controle químico realizado até então no Brasil, em 1978 a Embrapa-Trigo introduziu 14 espécies de parasitóides exóticos no Brasil, oriundos das mesmas regiões de origem dos pulgões (Ásia e Europa) (TAMBASCO, 1984; GASSEN, 1986). Nos insetários daquela Instituição foram reproduzidos os parasitóides e liberados nas regiões sul e centro-oeste do Brasil (GASSEN, 1999; SALVADORI; SALLES, 2002). Especificamente na região oeste do Paraná, segundo Cecconello* (comunicação pessoal) entre 1984 e 1985 foram introduzidas quatro espécies de parasitóides, Praon volucre, Aphidius colemani, A. uzbeskistanicus e Ephedron plagiator, havendo um trabalho paralelo com os produtores da região no sentido de implantarem medidas conservacionistas, visando auxiliar o estabelecimento dos parasitóides.

Os resultados foram desde o início muito animadores, sendo que no período de 1979 a 1981,

* Eng ${ }^{\mathrm{o}}$. Agr ${ }^{\mathrm{o}}$. Nelson José Cecconello, Palmas, TO, na época gerente do Dept ${ }^{\circ}$ Agronômico da Cooperativa Agroindustrial Lar.
Zúñiga-Salinas (1982) registrou em três áreas do Estado do Rio Grande do Sul baixa incidência populacional de pulgões. Da mesma forma, Tambasco (1984) confirmou a baixa incidência da praga entre 1980 a 1983, em Passo Fundo, RS., e, de acordo com Salvadori e Salles (2002), o controle populacional dos pulgões por microhimenópteros se mantém até hoje, devido à adaptação e estabelecimento dos mesmos nas regiões onde foram introduzidas.

No entanto, especificamente para a região oeste do Paraná, não se encontrou na literatura nenhum estudo sobre a avaliação do controle dos pulgões por microhimenópteros e assim, este trabalho foi realizado com o objetivo de atualizar tais informações, identificar as espécies e estudar a flutuação dos parasitóides e pulgões na cultura do trigo em Medianeira, PR.

\section{Material e Métodos}

O trabalho foi realizado na safra de inverno 2002, no município de Medianeira, PR. (416 metros de altitude, longitude $54^{\circ} 05^{\prime} 48^{\prime \prime} \mathrm{W}$, latitude $25^{\circ} 16^{\prime} 23^{\prime \prime}$ S), em uma área agrícola comercial, com 12,0 ha, na qual foi implantado o experimento em $1.500 \mathrm{~m}^{2} \mathrm{com}$ bordadura de no mínimo $30 \mathrm{~m}$ ao longo de toda a margem, semeada com trigo da variedade COODETEC 105, em 25 de abril de 2002. Adotouse espaçamento de $20 \mathrm{~cm}$ entre linhas com média de 58 plantas por metro linear e adubação com NPK 8 20-15, na quantidade de $173 \mathrm{~kg} / \mathrm{ha}$. $\mathrm{Na}$ área experimental não foi realizada nenhuma aplicação de qualquer produto fitossanitário durante todo o ciclo da cultura. O plantio foi realizado no sistema de plantio direto e a lavoura estava delimitada com áreas com plantio de milho "safrinha", trigo e vegetação nativa.

A população de afídeos foi avaliada semanalmente de abril a setembro de 2002, durante o desenvolvimento da cultura, a partir do estádio de um broto até o estádio de grão duro, tendo-se como base a escala Feekes (1941) para acompanhamento da fenologia da planta de trigo. 
Cada amostragem constituiu-se de 30 plantas cortadas rente ao solo, com auxílio de tesoura, acondicionadas em sacos plásticos transparentes e transportadas ao Laboratório de Biologia do Centro Federal de Educação Tecnológica do Paraná CEFET/ PR, Medianeira, PR, onde foram individualizadas, examinadas sob microscópio estereoscópico, sendo identificados e contabilizados os pulgões.

Os pulgões parasitados (múmias) encontrados e acompanhados dos dados de coleta foram isolados em cápsulas gelatinosas transparentes e mantidos em placas de Petri, sob condições ambiente e observadas diariamente para a verificação da emergência dos parasitóides. Estes foram encaminhados para o Prof. Dr. Ayres de Oliveira Menezes Jr. (Universidade Estadual de Londrina - UEL, PR), para identificação e depósito na coleção do Departamento de Agronomia da Universidade Estadual de Londrina, PR.

Os dados meteorológicos foram fornecidos pela Cooperativa Agroindustrial Lar, localizada a cerca de $800 \mathrm{~m}$ do local do experimento.

\section{Resultados e Discussão}

O número médio de pulgões encontrados não alcançou um pulgão por afilho, população esta considerada baixa visto que, de acordo com Silva e Ruedell (1983), a planta tolera até 5,1 pulgões/afilho, enquanto Salvadori e Salles (2002) caracterizam como dano econômico a incidência de 10 pulgões/ afilho do alongamento ao emborrachamento do trigo.

A espécie $R$. padi foi a mais abundante na área estudada (53\% de ocorrência), seguida de $S$. avenae e $R$. maidis, ambos com o mesmo percentual de ocorrência (19,5\%) e de M. dirhodum (8,0\%) (Figura 1). Os resultados diferem de levantamentos realizados por Pimenta e Smith (1976), nas regiões de Colombo e Ponta Grossa, PR, onde as espécies mais abundantes foram $S$. avenae e M. dirhodum. Zúñiga-Salinas (1982) citou as mesmas espécies como mais significativas para a Microrregião do Planalto Médio do Alto Uruguai e das Missões no estado do Rio Grande do Sul, ocorrendo ainda $R$. maidis, $R$. padi, S. graminum e $R$. rufiabdominali, porém em menor quantidade.

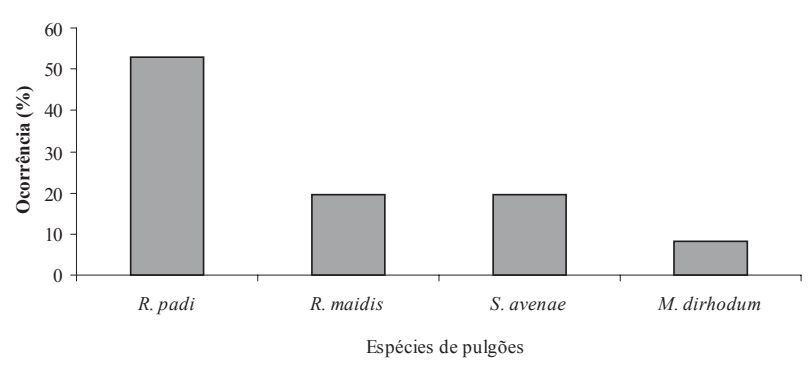

Figura 1. Composição da população de pulgões na cultura de trigo de abril a setembro de $2002 \mathrm{em}$ Medianeira, PR.

Com relação à época de ocorrência dos pulgões na lavoura, verificou-se que $R$. padi, $R$. maidis, $S$. avenae e $M$. dirhodum ocorreram na fase de alongamento do trigo, corroborando observações de Silva e Ruedell (1983), que verificaram maior incidência de $M$. dirhodum e $S$. avenae na fase final do alongamento da planta do trigo, em Cruz Alta, Rio Grande do Sul.

Em relação ao parasitismo, observou-se no mês de julho a presença de parasitóides e múmias de coloração pardo-clara com incremento na sua ocorrência a partir desta data, e conseqüente diminuição do número de pulgões ativos nas amostragens subseqüentes.

Foram encontradas apenas duas espécies de parasitóides, L. testaceipes e D. rapae (Hymenoptera: Braconidae) as quais foram responsáveis por $97,3 \%$ e $2,7 \%$ respectivamente, sobre os afideos $R$. padi, $S$. avenae, $R$. maidis e $M$. dirhodum. Ressalta-se que não foram encontrados registros na literatura sobre a predominância de parasitismo por L. testaceipes na região oeste do Paraná, embora a mesma tenha sido assinalada em outras regiões do Brasil, por Pimenta e Smith (1976), Rodrigues, Bueno e Bueno (2001), Salvadori e Salles (2002), Rodrigues, Bueno e Sampaio (2003), Carnevale, Bueno e Sampaio (2003). 
Segundo Salvadori e Salles (2002), esta espécie constava na lista de parasitóides liberados nas regiões produtoras de trigo do estado do Rio Grande do Sul. Provavelmente, ocorreu a dispersão deste parasitóide a uma distância de aproximadamente $1.200 \mathrm{~km}$ do local do presente estudo, com adaptação e estabelecimento nas lavouras da região.

Tal possibilidade pode ser confirmada pelo estudo de Rodrigues et al. (2001) que verificaram, em casade-vegetação, que este parasitóide apresentou boa capacidade de dispersão, deslocando-se cerca de três metros para buscar hospedeiros em um único dia. Sugeriram que, sem os limites da estufa, poderia percorrer distância maior em um único dia e ainda, que seria capaz de se desenvolver em várias espécies de pulgões de importância econômica. Essas características somadas à habilidade de encontrar os hospedeiros são atributos importantes desse agente para o controle biológico.

O parasitóide L. testaceipes é uma espécie Neártica, com ampla distribuição nas Américas do Norte, Central e do Sul (STARÝ; CERMELI, 1989). É de grande importância, visto que utiliza várias espécies de pulgões consideradas pragas nas culturas de milho, algodão, sorgo, citros e videira, como hospedeiros (STARÝ; CERMELI, 1989; RODRIGUES; BUENO, 2001).

De acordo com Starý e Cermeli (1989) L. testaceipes é um importante agente de controle biológico em diversas espécies de pulgões, como $S$. graminum (Rondani) e Aphis gossypii Glover. Além disso, Rodrigues e Bueno (2001) verificaram que $S$. graminum e A. gossypii mostraram-se adequados para o desenvolvimento de L. testaceipes, com taxas de parasitismo de 76 e $56 \%$, e emergência de 100 e $83 \%$, respectivamente.

Rodrigues et al. (2003) em estudo sobre a fertilidade de L. testaceipes em $S$. graminum, verificaram que o tempo médio entre o nascimento de uma nova geração deste parasitóide, foi de 11,13 dias, e enquanto que o tempo para a duplicação da população foi de 1,35 semanas, indicando que esse parasitóide tem condições de, em aproximadamente 10 dias, dobrar sua população.

Segundo Araiza et al. (1999), acompanhando a flutuação populacional de pulgões e seus inimigos naturais na cultura do trigo, no México durante quatro anos, verificaram que a atividade parasitária de $L$. testaceipes foi maior sobre $S$. graminum em todos os anos de estudo e mencionam que dentre os fatores ecológicos determinantes para a seleção do hospedeiro, a temperatura, o tamanho da colônia e a espécie de pulgão condicionam a eficácia do parasitóide, sendo que a baixa temperatura diminui a ação do mesmo.

Vale ressaltar que no estudo realizado, a temperatura média durante o período foi de $18^{\circ} \mathrm{C}$, fator que pode ter beneficiado a alta incidência e adaptação de L. testaceipes na cultura avaliada; mantendo baixos os níveis populacionais dos pulgões. A ausência do uso de inseticidas na área estudada, pode também ter contribuído para a manutenção das condições favoráveis aos inimigos naturais.

O controle dos pulgões foi considerado eficiente, pois a medida que a incidência de pulgões aumentou, gradativamente elevou-se o número de múmias (Figura 2). O parasitismo foi maior durante a floração do trigo, quando a incidência de múmias dos pulgões atingiu os índices mais elevados (65\%). Resultados semelhantes foram encontrados por Zúñiga-Salinas (1982) também em lavoura de trigo, no Rio Grande do Sul, onde os índices de parasitismo foram suficientes para manter as densidades de $S$. avenae e $M$. dirhodum muito abaixo dos níveis de dano econômico, não somente nos locais experimentais, como em áreas distantes da liberação. 


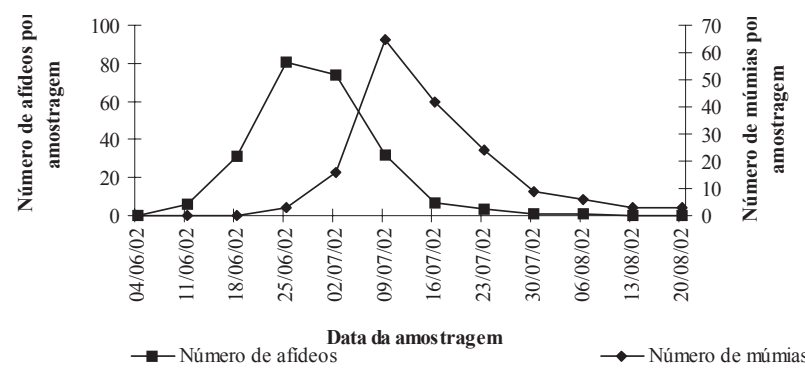

Figura 2. Flutuação populacional de pulgões e múmias de abril a setembro de 2002, na cultura do trigo em Medianeira, PR.

É importante ressaltar que nas proximidades da área experimental havia culturas de milho safrinha e vegetação nativa que podem ter influenciado na infestação de pulgões e ocorrência dos inimigos naturais no local do estudo.

No presente estudo, embora não se tenha avaliado a ação de outros inimigos naturais, verificou-se, em todas as amostragens, a presença de crisopídeos e coccinelídeos, citados por Gassen (1986), Salvadori e Salles (2002) como predadores de pulgões de trigo no Brasil, e que podem também ter contribuído para o controle biológico natural dos pulgões na área.

\section{Conclusões}

A espécie de pulgão na cultura do trigo mais abundante foi $R$. padi.

A população de pulgões foi maior na fase de alongamento da cultura do trigo.

Os parasitóides $L$. testaceipes e $D$. rapae foram eficientes no controle natural dos pulgões.

\section{Referências}

ARAIZA, M. D. S.; SOLÍS, E. S.; SALINAS, M. M. Fluctuación problacional de los áfidos del trigo y sus enemigo naturales en El Bajio, México. Manejo Integrado de Plagas y Agroecologia, México, v. 53, p. 19-27, 1999.

CARNEVALE, A. B.; BUENO, V. H. P.; SAMPAIO, M. V. Parasitismo e desenvolvimento de Lysiphlebus testaceipes (Cresson) (Hym.: Aphidiidae) em Aphis gossypii Glover e Myzus persicae (Sulzer) (Hem.: Aphididae). Neotropical Entomology, Londrina, v. 32, p. 293-297, 2003.

FEEKES, W. De tarwe en haar milieu. Verslagen Technische Tarwe Commissie, Groningen, v.17, p. 560-561, 1941.

GASSEN, D. N. Insetos associados à Cultura do Trigo no Brasil. Passo Fundo. Passo Fundo: EMBRAPA/CNPT, 1984.39 p. (Circular Técnica).

GASSEN, D. N. Parasitos, patógenos e predadores de insetos associados à cultura do trigo. Passo Fundo: EMBRAPA/CNPT, 1986. 86 p. (Circular Técnica).

GASSEN, D. N. Controle Biológico de Pulgões de Trigo no Brasil. Passo Fundo: EMBRAPA/TRIGO, 1999. 36 p. (Circular Técnica).

PIMENTA, H. R.; SMITH, J. G. Afideos seus danos e inimigos naturais em plantações de trigo (Triticum sp.) no Estado do Paraná. Curitiba: Ocepar, 1976. 175 p.

RODRIGUES, S. M. M.; BUENO, V. H. P. Parasitism Rates of Lysiphlebus testaceipes (Cresson) (Hym.: Aphidiidae) on Schizaphis graminum (Rond.) and Aphis gossypii Glover (Hem.: Aphididae). Neotropical Entomology, Londrina, v. 30, p. 625-629, 2001.

RODRIGUES, S. M. M.; BUENO, V. H. P.; BUENO DE, J. S. $\mathrm{S}$. Desenvolvimento e avaliação do sistema de criação aberta no controle de Aphis gossypii (Glover) (Hem:Aphididae) por Lysiphlebus testaceipes (Cresson) (Him.: Aphidiidae) em Casa de Vegetação. Neotropical Entomology, Londrina, v. 30, p. 625-629, 2001.

RODRIGUES, S. M. M.; BUENO, V. H. P. e SAMPAIO, M. $\mathrm{V}$. Tabela de vida de fertilidade de Lysiphlebus testaceipes (Cresson, 1880) (Hymenoptera, Aphidiidae) em Schizaphis graminum (Rondani, 1852) (Hemiptera, Aphididae). Revista Brasileira de Entomologia, São Paulo, v. 47, p. 637-642, 2003. 
SALVADORI, J. R.; TONET, G. E. L. Manejo Integrado dos Pulgões de Trigo. Passo Fundo: EMBRAPA/CNPT, 2001. 52 p. (Circular Técnica).

SALVADORI, J. R.; SALLES, L. A. B. Controle Biológico dos Pulgões do Trigo. In: PARRA, J. R. P.; BOTELHO, P. S. M.; FERREIRA, B. S. C.; BENTO, J. M. S. (Ed.) Controle Biológico no Brasil: parasitóides e predadores. São Paulo: Manole, 2002. p. 427-447.

STARÝ, P.; CERMELI, M. Parasitoides (Hym.:Aphidiidae) de áfidos en plantas cultivadas de Venezuela. Boletin de Entomologia Venezolana., Caracas, v. 5, n. 10, p. 77-80, 1989.
SILVA, M. T. B. da.; RUEDELL, J. Efeito de seis níveis populacionais de pulgões na fase vegetativa do trigo. In: REUNIAO NACIONAL DE PESQUISA DE TRIGO, 13., 1983, Cruz Alta. Anais...Cruz Alta, 1983.

TAMBASCO, F. J. Determinação de níveis de dano de pulgões em trigo. Passo Fundo: EMBRAPA/CNPT, 1984, 113 p. (Circular Técnica).

ZÚÑIGA-SALINAS E. S. Controle biológico dos afideos do trigo (Homóptera: Aphididae) por meio de parasitóides no Planalto Médio do Rio Grande do Sul, Brasil. 1982. Dissertação (Mestrado) - Departamento de Entomologia, Universidade Federal do Paraná, Curitiba, 1982. 